\title{
Semantic Web Ontologies based Knowledge Management Framework for IT Service Management
}

\author{
Varsha Deb, Vasudha Vashisht, Nidhi Arora
}

\begin{abstract}
Technology driven organisations are investing hugely in training and knowledge enrichment of their employees. This is due to the fact that knowledge is now considered as an asset by organisations. Additionally, with emerging technologies, organisations are also spending heavily in Information and Communication Technology (ICT) to enhance their internal operations and processes. Among the various internal processes, Knowledge Management is an area which has been there since many years but when it is about the application of latest technology and innovations for Knowledge Management practices, there are huge opportunities. This paper presents an analysis of various KM frameworks available for different domains and based on current state and limitation identified, it proposes a Semantic Web Ontology based Knowledge Management System for IT Service Industry
\end{abstract}

Index Terms: Knowledge Base, Knowledge Management System, Knowledge Reusability, Ontologies, OWL, Protégé, Semantic Web

\section{INTRODUCTION}

The ability of a service organisation to deliver services to the customer heavily depends on its ability to understand the customer's business scenarios and respond to them promptly. With tough competition among service providers, they strive for having various ways and means to respond to customers' requirements as promptly as possible. This requires them to reuse the exiting knowledge and information about the customers and their past requirements. These service providers also maintain a reusable knowledge repository called Knowledge Base (KB) on various tools to search readymade solution and prompt actions.

Today when the world is moving towards knowledge based economy, it is imperative that IT service organisations too move towards knowledge reuse with the help of latest AI enabled tools. With the advent of technology and various business processing tools, industries are moving from being person dependent to having the customers' requirements /requests being logged and processed by using various Information Technology Service Management tools (ITSM Tools), such as ServiceNow, JIRA, SolarWind, etc. Most of these tools are equipped with converting various solutions, provided while resolving requests, to knowledge.

To provide better understanding about working of an IT service organisation, Fig 1 shows a typical lifecycle of a

Revised Manuscript Received on July 5, 2019.

Varsha Deb, Amity University, NOIDA, UP, India,

Vasudha Vashisht, Amity University, NOIDA, UP, India

Nidhi Arora, GD Goenka University, Gurugram, Harayana, India request raised by a user to an IT service helpdesk.

It depicts a user, facing an issue, or an Incident, in application or infrastructure service(s), contacts the helpdesk engineer, called Level 1, who analyses the user's issue and accordingly logs and tries to resolve by referring to the $\mathrm{KB}$. If the solution is not available in the $\mathrm{KB}$, the engineer troubleshoots and tries resolving the incident. In the event the incident is not resolved, it is passed to the Level 2 expert engineers after appropriate updates and prioritization. At this level too if the incident is not resolved, it is passed to Level 3 experts for resolution.

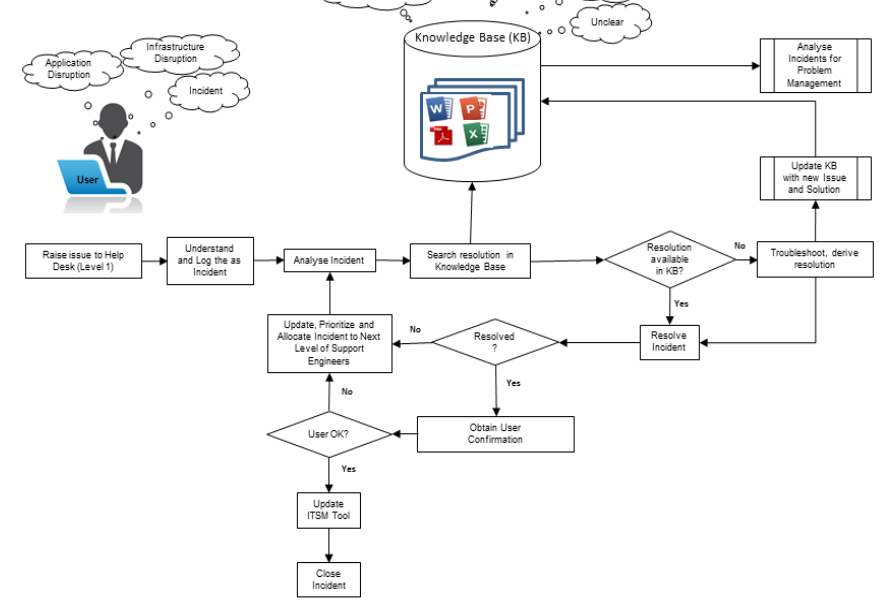

Fig. 1. Lifecycle of a request

At each level, the KB is referred to, for available solution. Every time an issue occurs, engineers are expected to update the solution in the KB to keep it up-to-date. Additionally, obtaining confirmation from user is necessary to ensure the issue has been fixed at user's side. In this lifecycle, it is very much evident that if an issue is resolved at Level 1, it results in early restoration of user service as well lesser turnaround time for the service provider; offering win-win situation for both. Thus, one of the key activities that offers value to the customer is resolving more and more requests by Level 1 or Level 2 teams, which is called left shifting the work in the team. The work that cannot be processed by Level 1 team should be passed to the next levels. However, in many cases, the requests which should have been processed by the Level 1 team are also passed to the next level teams, who are costlier resources than the upstream. 
The reasons for this handoff are majorly related to people skill and knowledge, and insufficient, scattered, obsolete and ambiguous knowledge available in the $\mathrm{KB}$, which has been established by the authors in their previous paper [1]. The Table 1 lists various reasons for the non-optimum usage of KMS.

TABLE I. FACTORS IMPACTING NON-OPTIMUM USAGE OF KMS

\begin{tabular}{|r|l|l|}
\hline \multicolumn{1}{|l|}{ S. No } & Factors hindering the usage of KB & \% Response \\
\hline 1. & $\begin{array}{l}\text { To the point and concise information not } \\
\text { available }\end{array}$ & $20 \%$ \\
\hline 2. & Time consuming search & $19 \%$ \\
\hline 3. & Required information/resolution not available & $18 \%$ \\
\hline 4. & Information scattered on various tools & $13 \%$ \\
\hline 5. & KB not updated has obsolete information & $13 \%$ \\
\hline 6. & $\begin{array}{l}\text { Not needed anymore; as the solutions } \\
\text { available are now known }\end{array}$ & $8 \%$ \\
\hline 7. & Lack of Knowledge on usage of KB & $6 \%$ \\
\hline 8. & KB not established & $2 \%$ \\
\hline
\end{tabular}

The following sections will first details the importance of knowledge andknowledge management, followed by review of present work done in this area.

\section{IMPORTANCE OF KNOWLEDGE AND KNOWLEDGE MANAGEMENT}

In the context of organisations, Knowledge Management can be defined as the practice of collecting knowledge from various heterogeneous sources and subject matter experts; organizing and storing them for the purpose of reuse. The key objective is to ensure that the right person has the right knowledge, at the right time, to deliver the services required by the business, resulting in faster turnaround time and reduction in quality issues.

However, in addition to facing the challenges as listed in section 1 above, the industry is facing the challenge of knowledge loss due to attrition, retirement, and internal job rotation of people. A strong Knowledge Management System is capable of resolving such issues. In [2] the authors have highlighted the importance of managing knowledge as critical part of business. This paper mentions that not managing the knowledge of the organisation properly is a critical risk and a major threat to business continuity in case of disaster. The paper stresses that the Knowledge Management should also be considered while planning for the business continuity for any service, while citing the unfortunate incident of 9/11 in the USA, which shattered many organisations and put a halt to business.

To strengthen this argument and the necessity to have good $\mathrm{KB}$, a recent survey done by Gartner [3], presents that there is a need to focus on amalgamation of information and knowledge, which is available discretely in various formats and tools to deliver value to the customers. Current KM tools available in these organisations are focusing more on providing reusable solutions to helpdesk, which is also termed as Level 1 team, not to the support engineers (called Level 2 and Level 3) who are involved in bug fixing and configuration

of applications/infrastructure components.

The following section 3 , first presents a brief introduction to ontologies and its importance in knowledge management, followed by literature review in the field of knowledge management and usage of ontologies. Based on the literature review, section 4 presents a proposed system that helps overcoming the issues discussed in previous sections.

\section{ONTOLOGIES FOR KNOWLEDGE MANAGEMENT}

The concept of Ontologies has been borrowed from a branch of Philosophy; it entails the study of existing or being. Ontologies is a concept of semantic web that enables the development of algorithms to derive inferences based on certain facts and ensuring consistency [4].

Ontologies act as repository to organize knowledge and information based on common vocabulary. They optimize knowledge retrieval and exchange of knowledge and support reuse of knowledge with reasoning and inferential ability to provide more insight on existing knowledge. Ontologies can be used to achieve improved efficiency in information retrieval, processing and presenting structured, partially structured and unstructured data in machine readable format [5] [6].

A report by IDC suggests that analysis of data is limited to only $1 \%$ of data available. Garters reports also predict $800 \%$ increase in the volume of data, out of $80 \%$ being unstructured [7].

Section 2.1.2 of [29] cites two benefits of ontologies over traditional approaches by stating that, ontologies support to join information which is otherwise available in silos. They offer additional background knowledge that can help a novice to query and extract information based on their view point.

These benefits offered by ontologies also offers solution for many of the challenges faced by IT service organisations, viz. 1, 2, 3, 4 and 8 cited in the Table 1. Overcoming these causes, will address the $72 \%$ of the factors listed in the table; thereby increasing the use of $\mathrm{KB}$.

\section{A. Literature review to assess present work and implementation}

\section{1) Some Statistics}

In past, a lot of work has been done on establishing various knowledge management practices. The following section covers the various frameworks proposed by authors for knowledge management in a variety of domains. Tounderstand the work done in this area, we performed the literature search on IEEE using the key words "Knowledge Management Framework" and "Ontologies". The search produced a total of 919 artifacts from the year 1992 to 2018 . The column chart presented in Fig 2. shows a trend of artifacts published.

Though there is a decrease in the work being done in this area, still the problems that industry is facing needs to be addressed. 


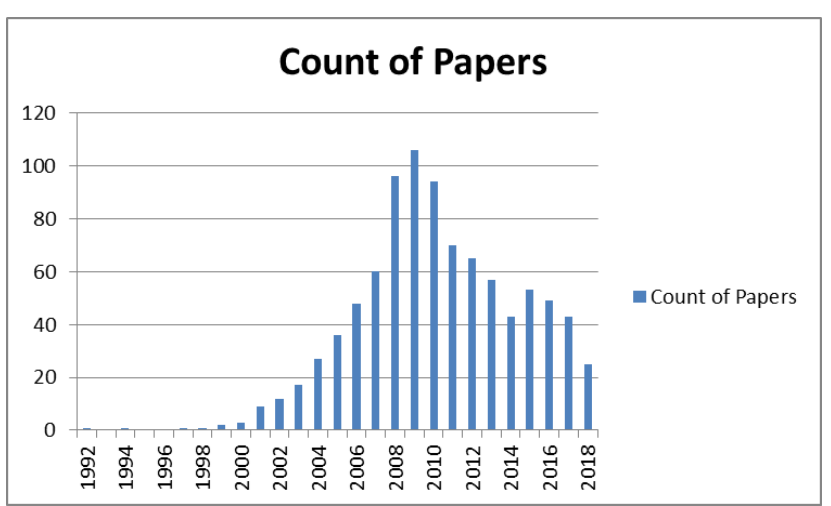

Fig. 2. Count of Papers Published from 1992 to 2018

\section{2) Ontologies for IT Services.}

Based on the survey conducted in the previous paper [1] of authors, it was found that only $2 \%$ of IT Service Management organisations are using latest technologies for KB. Others use various traditional ways to store the knowledge such as MS Excel, Web pages, MS Word or PDF Documents; some use database based solutions too.

In the event of missing information, database uses the concept of Closed World Assumption (CWA) and returns a ' 0 ' if the search string is not found; the ontology works using the concept of Open World Assumption (OWA) and considers missing information as 'unknown' on the premise that this information may be available at another repository. The purpose of creating a database is to store and retrieve information when needed; the ontologies focus on adding meaning and comprehension. Additionally, as the information is stored in various heterogeneous unstructured formats and distinct sources, integrating all the information to derive knowledge is another challenge is posed today [20]. Knowledge Management not only aims towards preserving past knowledge but also generating new knowledge; it is pivotal to maintain the existing knowledge and populate the $\mathrm{KB}$ with new knowledge.

Various tools that are used by the service providers to log and track the requests also have capability of storing the knowledge. However, integrating the offline knowledge available in various structured, partially structured and unstructured formats is a humongous task; the same issue has been cited as one of the factors that discourage service providing agents to use $\mathrm{KB}$.

3) Using ontologies for ITSM.

Considering the benefits of ontologies and the limitation of traditional way of storing and retrieving knowledge; an ontology based KM mechanism will help the ITSM. To understand the availability of KM systems for ITSM domains that helps the support engineers in resolving the request, a literature availability search was performed. Based on the literature searched on IEEE for the keywords "ITSM and Ontologies/Ontology a very limited papers have been published. However, the latest Gartner report [3] and survey results presented by authors stress the need to relook at the problem and solutions proposed up till now. Additionally, ITSM that constitute majority of business for IT industry in India today strengthens this need.

The following section provides a brief view of the papers

found based on the above search criteria.

[22] This paper proposes a conceptual model of domain ontology for IT service configuration management. However the scope of this ontology can be extended to information technology infrastructure library or ITIL Framework. ITIL is a set of service management standard library for IT industry. [23] proposes the integration of ontologies for software engineering and IT service management domain. This paper also mentions about inclusion of ITIL framework while designing the ontologies. This can be enhanced to generate an end to end framework that optimizes knowledge retrieval from ontologies and automatic updates. [24] This paper presents Wiki and semantic ontologies based monitoring system for monitoring for infrastructure components. The scope of this paper is limited to the monitoring services. The paper does not cover much information on information retrieval and updation.

[25] This paper presents semantic based structure for IT service management processes to design and maintain ITIL processes. The work covers the different phases of ITIL framework without providing much detail on information related to various components of service operations, like incident and problem management.

[26] presents a method, DEMO, to identify the various services offered by a service provider for service portfolio and catalog management with a limited or no coverage of other areas of IT Service Management. [27] proposes ontology based knowledge representation method for ITSM for IT operations and maintenance and maps it to knowledge base. It applies the case knowledge management to facilitate knowledge sharing and retrieval, enhance the recall and precision. With the help of concept sharing mechanism, the search resolves redundancy and consistency issue. This paper does not cover how the effectiveness of the proposed work can be evaluated. There is a scope to enhance this model by designing the model based on ITIL, which will ensure an end-to-end availability of knowledge for entire service lifecycle, starting from Service Transition to Service Operations till Service Closure.

The following section briefly covers work done in other domain using ontologies for knowledge management.

4) Ontology's implementation in various domains.

The following section presents review of some related KM frameworks established using ontologies in various domains.

COMANCHE SCM framework is a knowledge management framework for Software Configuration Management (SCM) based on ontologies to represent knowledge required to perform configuration management activities. This uses Description Logic Reasoning for knowledge inference and Protégé OWL Ontology editor to manage the ontologies. Reasoner used in the example is $\mathrm{FaCT}++$. Framework is composed of following components: Attribute Provider, Context Provider, Knowledge Manager, Attribute and Context Knowledge Base, Knowledge Registry. This framework organizes information about SCM attributes and infers which software service is to be installed on a given user devise [8]. 
KM Framework presented by Simone Maccanti et al. [14] for Software Reuse during the Software Development to help software developer and engineers for IT software development domain. The framework proposes to host all heterogeneous and unstructured data in a data warehouse. It further proposed the use of ETL tools to transforming the data in structured format and load in the Knowledge Base. The proposed format to load the data in Knowledge Base is a markup language. It mentions about usage of rules and patterns for information extractions, and in case the required knowledge is not found in the knowledge base, the framework suggests searching in other databases.

Semantic Web Ontologies have been used in agricultural domain too for managing the knowledge. This ontology uses FOL (First Order Logic) and OWL (Web Ontology Language); Protégé Ontology Editor to create Ontologies and SPARQL to query the information from Ontologies. However, the reasoning and inferencing functionality by Protégé is used. This ontology consolidates the scattered information available to answer the generic questions related to fertilizers [9]. Technology based framework for KM on Tourism Crisis Management in Travel and Tourism domain. The objective of this work is to enable fact based decision making using the KMS to be able to respond swiftly and recover with minimal impact in the event of eventualities. This framework has used databases to store the data and information in the form of digital data, with a use of an information retrieval engines. It uses Wikis as the knowledge creation method. Web technologies like PHP and XML have been used to create user interface to access the knowledge. It uses the document management system to manage the knowledge documents. It also makes use of data mining and knowledge discovery technique to transform data into information.

Semantic Web has been applied for development of three tier architecture of knowledge management system for Government Repository that is available in scientific documents, papers, research projects and technical projects. It proposes creation of knowledge repositories using Oracle XML database. This paper also presents a method for extraction of semantic features from documents to be added in the knowledge base in the form of XML taxonomies and XML metadata. [11]

[12] presents work towards understating the impact of Social Media Network in healthcare Knowledge Management System. This work proposes Ontological-based Social Media Supported Framework (SMKMS) for the healthcare domain. They used Protégé and top-braid composer; and OWL as the language. They also use SPARQL queries. The researcher further developed RDF version of ontologies using Java Jena Ontology API. Additional implementation of FOAF (Friend of a Friend) user profile description technique enabled to establish connection among various users (health professionals).

The concept has been used in construction industry [13], where Ontology based KMS has been designed using Protégé and OWL. Pellet reasoning engine is used for semantic reasoning. A Java based knowledge extraction module was created for knowledge retrieval. Another framework for the
Zhiyang Jiaa et al. [10] proposed an AI and Web

same domain has been built to improve energy efficiency of buildings, a platform MonitoringLab has been introduced using Ontologies and intelligent filtering methods with the purpose to integrate ontologies and filtering method.

KM frameworks have also been established to study the problem of obesity in children with a noble objective to prevent the problem with the use of Blooms technique for healthcare domain. Imbalance data is one of the major challenges for Knowledge Discovery phase, clustered ontology based knowledge management framework using the Simple Hybrid Sampling Approach and Frequent Pattern Mining algorithm is useful[15][16]. Ontology based framework have also proved helpful in Product organisations for knowledge representation [18]. [19] proposes a framework to enable informal knowledge sharing for Agile Software Development teams using real-time collaboration environment. However it does not mention about techniques used for knowledge acquisition or retrieval.

\section{Proposed System}

Based on the study presented in sections above, it can be concluded that the work done in this area can further be extended for the use of industry. This section proposes a Semantic Web ontology based solution for end to end knowledge management cycle consisting of knowledge creation, knowledge retrieval and knowledge updation[17].

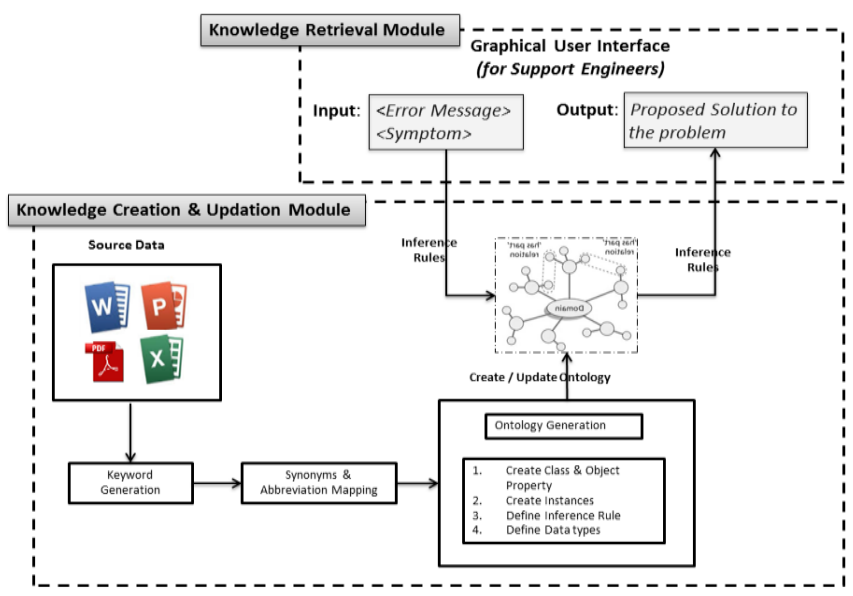

Fig. 3. Proposed System

Proposed system consists of two modules, first is Knowledge Creation and Updation Module for ontology creation and updation, and second as Knowledge Retrieval Module for knowledge extraction by the support engineers/agents.

For any IT Service, most of the information to troubleshoot an issue is documented in SOPs, which can be in the form of MS Word, MS PowerPoint and Adobe PDFs, the NLP based ontology generation module scans these files and enumerates the key terms. The extracted terms with the help of SME are then defined as class, class hierarchy, properties and their instances. For this scenario, the classes Configurable_Item, Error_Message, Symptoms and Proposed_Solution are created. 
The class Configurable_Item contains subclass as server, network, end_points. The class Configurable_Items has object property as hasOwner, hasVersion, hasOS, hasMemory, hasAvailability, hasEndOfLife. The class Symptom's objective is to have abnormal symptoms of the infrastructure components, each referred as a CI. This is related to class Configurable_Item using the object property hasSymptom. Another class error_message is related to Configurable_Item using hasError object property. Inference rules can be defined based on Symptom and Error_message classes and information from Proposed_Solution class is extracted, giving the proposed solution to the agent quickly.

The solution can later be enhanced by deploying bots that can directly pass the input from queries/request to the $\mathrm{KB}$ and retrieve the information from the ontology. To keep the KB updated, automatic generation of more instances of error message, solutions, symptoms need to be updated. This can be achieved by taking the input from the service management tool that has the information of the query or request, along with details of issue and the solution that is entered by the agent on resolution of the query / request. This feature will help to ensure all new types of information which is currently not available gets uploaded in the KB. To measure the effectiveness of proposed solution, the agent can mark the issue that has been solved using the KM and later on a count will help.

\section{CONCLUSION}

This paper presented a Semantic Web Ontologies based framework for management of knowledge for IT Services. This framework caters to the need of knowledge users who handle repetitive type of work. The automatic generation of ontology based $\mathrm{KB}$ will help the users by offering reusable solution to the variety of problems faced by them while troubleshooting. This will enables the IT Service providers to monitor the health of various infrastructure components being managed, take appropriate actions based on the past information available in the $\mathrm{KB}$ and monitor the progress of health of infrastructure components.

\section{REFERENCES}

1. V. Deb, V. Vashisht and N. Arora, "An Analytical Approach to Improve the Effectiveness and to Assess Current Technological Trends \& Challenges of Knowledge Management System," 2018 8th International Conference on Cloud Computing, Data Science \& Engineering (Confluence), Noida, 2018, pp. 14-15.doi: 10.1109/CONFLUENCE.2018.8443037

2. Knowledge Management: The Missing Element in Business Continuity Planning Denise Johnson McManus Wake Forest University, USA, Charles A. Snyder, Auburn University, USA, DOI: 10.4018/978-1-59904-933-5.ch211

3. Gartner Report: 2018 strategic roadmap for 2018; Published: 5 April 2018; ID: G00346258; Chris Matchett, Ed Holub, Colin Fletcher, Hank Marquis, Kenneth Gonzalez, Roger Williams, Siddharth Shetty, Robert Naegle

4. Using Ontologies for Knowledge Management:An Information Systems Perspective;Igor Jurisica, John Mylopoulos, Eric $\mathrm{Yu}$;University of Toronto, Toronto, Ontario, Canada; Annual Conference of the American Society for Information Science, Washington, D.C., November 1-4, 1999.

5. Vasudeva Varma; Chapter 2; USE OF ONTOLOGIES FOR ORGANIZATIONAL KNOWLEDGE MANAGEMENT AND KNOWLEDGE MANAGEMENT SYSTEMS; Ontologies: A Handbook of Principles, Concepts and Application in Information Systems; http://www.springer.com/978-0-387-37019-4

6. Agnieszka Konys / Procedia Computer Science 126 (2018) 2208-2218 2209

7. https://www.netowl.com/2017/08/11/80-worlds-data-unstructured-ent ity-extraction-must

8. Ploskas, Nikiforos \& Berger, Michael \& Zhang, Jiang \& Wintterle, G.-J. (2008). A Knowledge Management Framework for Software Configuration $\quad$ Management. $\quad 593 \quad$ - 598. 10.1109/COMPSAC.2008.106.

9. Malik Nidhi, Sharan Aditi, Semantic Web Oriented framework for Knowledge Management in Agriculture Domain, International Journal of Web Applications Volume 8 Number 3 September 2016

10. Zhiyang Jiaa, Yiyin Shia, Yuan Jiab, Ding Lia, A Framework of Knowledge Management Systems for Tourism Crisis Management; International Workshop on Information and Electronics Engineering (IWIEE); Procedia Engineering 29 (2012) 138-143; doi:10.1016/j.proeng.2011.12.683

11. Edgar Tello-Leal, Ana B. Rios-Alvarado, Alan Diaz-Manriquez "A Semantic Knowledge Management System for Government Repositories", in 2015 26th International Workshop on Database and Expert Systems Applications

12. The impact of social media networks on healthcare process knowledge management (using of semantic web platforms); Abid Ali Fareedi; Syed Hassan; 2014 14th International Conference on Control Automation and Systems (ICCAS 2014) Pages: 1514 - 1519

13. L.Y. Ding, B.T. Zhong, S. Wu, H.B. Luo, Construction risk knowledge management in BIM using ontology and semantic web technology, Safety Science 87 (2016)

14. Simone Maccanti, Jameela Al-Jaroodi, Arif Sirinterlikci; Knowledge Management Framework for Software Reuse; 2016 IEEE 40th Annual Computer Software and Applications Conference; DOI 10.1109/COMPSAC.2016.147

15. Raslapat Suteeca, Prompong Sugunnasil; A Knowledge Management Framework for Studying the Child Obesity; 2016 International Conference on Industrial Engineering, Management Science and Application (ICIMSA)

16. Sally M. El-Ghamrawy; A Knowledge Management Framework for imbalanced data using Frequent Pattern Mining based on Bloom Filter; 2016 11th International Conference on Computer Engineering \& Systems (ICCES)

17. S. Mohapatra et al., Designing Knowledge Management-Enabled BusinessStrategies, Management for Professionals, DOI 10.1007/978-3-319-33894-1_2

18. Zhang Dongmin, $\mathrm{Hu}$ Dachao, $\mathrm{Xu}$ Yuchun; A Framework for Ontology-based Product Design Knowledge Management; 2010 Seventh International Conference on Fuzzy Systems and Knowledge Discovery (FSKD 2010)

19. R. K. Kavitha, M. S. Irfan Ahmed; A Knowledge Management Framework for Agile Software Development Teams; 2011 International Conference on Process Automation, Control and Computing

20. Michal Sir, Zdenek Bradac, Petr Fiedler, Ontology versus Database, IFAC-PapersOnLine, Volume 48, Issue 4, 2015, Pages 220-225, ISSN 2405-8963,https://doi.org/10.1016/j.ifacol.2015.07.036.

21. Leung, Nelson K. Y. \& Lau, Sim \& Fan, Joshua. (2007). An Ontology-Based Knowledge Network to Reuse Inter-Organizational Knowledge.

22. G. Baioco, A. C. Monteiro Costa, C. Z. Calvi and A. S. Garcia, "IT service management and governance modeling an ITSM Configuration process: A foundational ontology approach," 2009 IFIP/IEEE International Symposium on Integrated Network Management-Workshops, New York, NY, 2009, pp. 24-33. doi: 10.1109/INMW.2009.5195930

23. M. Valiente, E. García-Barriocanal and M. Sicilia, "Applying Ontology-Based Models for Supporting Integrated Software Development and IT Service Management Processes," in IEEE Transactions on Systems, Man, and Cybernetics, Part C (Applications and Reviews), vol. 42, no. 1, pp. 61-74, Jan. 2012. doi 10.1109/TSMCC.2011.2132717

24. F. Kleiner, A. Abecker and S. F. Brinkmann, "WiSyMon: Managing Systems Monitoring Information in Semantic Wikis," 2009 Third International Conference on Advances in Semantic Processing, Sliema, 2009, pp. 77-85. doi: 10.1109/SEMAPRO.2009.13 
25. M. Sarnovsky and K. Furdik, "IT service management supported by semantic technologies," 2011 6th IEEE International Symposium on Applied Computational Intelligence and Informatics (SACI), Timisoara, 2011, pp. 205-208. doi: 10.1109/SACI.2011.5873000

26. C. Mendes, J. Ferreira and M. M. da Silva, "Using DEMO to Identify IT Services," 2012 Eighth International Conference on the Quality of Information and Communications Technology, Lisbon, 2012, pp. 166-171. doi: 10.1109/QUATIC.2012.67.

27. Xin Zhang, Xingyu Chen, Shaoyong Guo and Zhiqiang Zhan, "Ontology-based ITSM knowledge representation research," 2010 International Conference on Advanced Intelligence and Awarenss Internet (AIAI 2010), Beijing, China, 2010, pp. 230-235. doi: 10.1049/cp.2010.0759

28. G. Kim and D. Lee, "Intelligent Health Diagnosis Technique Exploiting Automatic Ontology Generation and Web-Based Personal Health Record Services," in IEEE Access, vol. 7, pp. 9419-9444, 2019;.doi: 10.1109/ACCESS.2019.2891710

29. Applications of Ontologies in Software Engineering; Hans-Jörg Happel and Stefan Seedorf; Semantic Scholar; Published 2006

30. Natalya F. Noy and Deborah L. McGuinness, Ontology Development 101: A Guide to Creating Your First Ontology

\section{AUTHORS PROFILE}

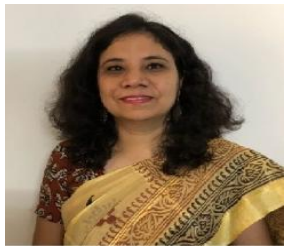

Varsha Deb is a research scholar at Amity University, NOIDA, UP, India and an IT professional. Her area of research is Knowledge Management and Artificial Intelligence. She has an overall experience of 21 years in IT MNC in the field of Software Development, Software Maintenance, quality assurance and management of ISO and CMMI certifications.

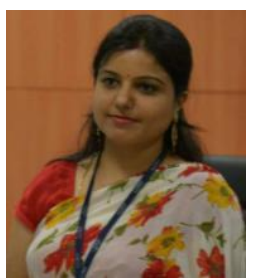

Dr. Vasudha Vashisht is currently working with Amity University, Uttar Pradesh as an Associate Professor and Researcher. She has completed her $\mathrm{Ph} . \mathrm{D}$. in the area of Artificial Intelligence. She has authored more than 25 Research Publications in National and International Conferences \& Journals. She has filed two Patents for BCI research work and received the copyright of her research work from Govt. of India. She has also received an award of Industrial Project. She is Microsoft certified Faculty Fellow, Reviewer and Editorial Board Member of reputed International Journal(s) She has also received many Letters of Appreciation for Academic, Research and Social works. She has organized and attended many Workshops \& Training Programs, Seminars etc. She has more than 15 years of experience in university teaching \& research.

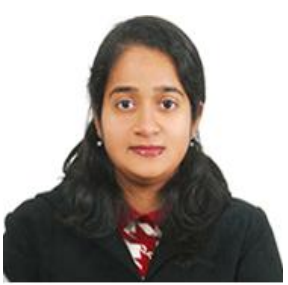

Dr. Nidhi Arora is a $\mathrm{PhD}$. in Information Retrieval from INHA University, South Korea. Her dissertation work focused on designing a ranking algorithm to produce top-k search results for keyword query on data graphs. This work has been published in top conferences and journals such as DEXA, DASFAA, Expert Systems With Applications (ESWA) and New Generation Computing. She has mentored undergraduate projects such as Address Cleansing using Condition Random Fields and Subjective Answer Analyzer using LSTM and Neural Networks. 